\title{
In vitro Photo-Controlled Drug Release System Based on Amphiphilic Linear-Dendritic Diblock Copolymers; Self-Assembly Behavior and Application as Nanocarrier
}

\author{
Hassan Namazi ${ }^{1,2,3}$ and Saeed Jafarirad ${ }^{1,3}$ \\ ${ }^{1}$ Research Laboratory of Carbohydrates and Biopolymers, Faculty of Chemistry, University of Tabriz, Tabriz, Iran. \\ ${ }^{2}$ Research Center for Pharmaceutical Nanotechnology (RCPN), Tabriz University of Medical Sciences, Tabriz, Iran. \\ ${ }^{3}$ Research institute for fundamental sciences (RIFS), University of Tabriz, Tabriz, Iran. \\ Received, January 12, 2011; Revised May 2, 2011; Accepted, May 4, 2011, Published May 5, 2011.
}

\begin{abstract}
Purpose. A simple type of photoresponsive amphiphilic linear-dendritic diblock copolymer has been synthesized and investigated for its ability to act as a drug carrier. These structures contain hydrophilic polyethylene oxide monomethyl ether (PEOM) as hydrophilic block and carbosiloxane dendritic branches as hydrophobic block grafted by two and six Magneson II as azo chromophore, PEOM-Azo, 2 and PEOM-Azo, 6 respectively. Self-assembling of the amphiphilic macromolecules of PEOM-Azo, 2 and PEOM-Azo, 6, leads to the formation of their micellar aggregates in aqueous media. Method. Their micellar properties such as critical micelle concentration (CMC), aggregation number and thereby total numbers of azo-groups in each micellar aggregates were determined. Also, they were characterized by TEM, SEM and DLS. Results. The unloaded aggregates examined under UV light $(366 \mathrm{~nm})$, which were observed to be smaller than $300 \mathrm{~nm}$. The release patterns of the encapsulated drug molecule from these aggregates were investigated as regulated by the PEOMAzo, 2 and PEOM-Azo, 6 systems in trans and cis forms. Conclusion. A comparison of the release behavior of the loaded PEOM-Azo, 2 and PEOM-Azo, 6 systems indicated that the release rate of the encapsulated active molecules from the carriers was slower when the azo moieties were in trans form as compared to that the azo in the cis form. The in vitro release behavior of drug from these polymeric systems represents potential of the carriers for controlled drug delivery.
\end{abstract}

This article is open to POST-PUBLICATION REVIEW. Registered readers (see "For Readers") may comment by clicking on ABSTRACT on the issue's contents page.

\section{INTRODUCTION}

The recent few years have witnessed increased interests in the development of controlled drug delivery systems by amphiphilic macromolecules. Briefly, an ideal controlled release possesses three steps including; (i) the effective encapsulation of the drug by polymeric micelles (1) (ii) a site-specific transportation of the drug (2) (iii) and finally, the polymeric micelle "opens the door" to release the bioactive molecule (3). For each of the three steps there have been much attempts directed toward developing the best strategies. In deed, the first and third steps only participate in release of drug effectively. In the first step, a stable encapsulation of the drug, which is crucial to the whole of delivery, could also affect the drug release from the micelle to some what. Also, in the third step, in order to release the drug in a controlled way, the micelle should be conformationally changed. Therefore, it is rational to think about micelles with ability to react to external stimulus. Most of research on stimuli-responsive polymeric micelles deals with $\mathrm{pH}(4,5)$ and temperature (6). Recently, light irradiation is considered as the most reliable external stimulus owing to involving only photochemical processes, which are energy-saving and easily controllable (7). The most attractive aspect of this method is the offering control over when and where the release of loaded agents takes place.

Corresponding Author. Hassan Namazi, Research Laboratory of Carbohydrates and Biopolymers, Faculty of Chemistry, University of Tabriz, Tabriz, Iran. namazi@tabrizu.ac.ir 
Accordingly, it is predictable that, in the future researchers could design a special kind of light source as a portable kit which can be manipulated from outside of the body so that the release could take place when illumination is applied only to the region exposed to light. However, despite of interesting aspects of photo-responsive materials due to the possibility of developing materials sensitive to electromagnetic radiation (mainly in the UV, visible and near-infrared range), which can be applied on demand at well delimited sites of the body, some drawbacks are present.

For example, does the near-UV light, which will trigger the drug release, effectively overcome skin and tissue thickness to successfully reach the micellar systems, assuming the system reached a hypothetical disease target?

It has been shown that, by grafting photoresponsive chromophores to the self-assembled structures of amphiphilic macromolecules, those interesting properties can be further extended to new areas of material design (8). For instance, they were underwent a reversible changes in the physical properties such as the polarity, hydrophobicity, conformation and morphology of the micellar aggregates $(9,10)$. Amongst the photoresponsive units azobenzene family mesogens, in particular, has attracted special attention due to their medical applications, for example, as colon-specific therapeutic agents (11). It is as a result of high enzymatic degradation of azo moiety by azoreductases produced by microflora of the gastrointestinal tract (12). However, most research works in these fields have been exploring smallmolecule systems containing azobenzene photochromic units (13). Furthermore, construction of self-assembled supramolecular structures by using
$\mathrm{N}=\mathrm{N}$ trans-cis photoisomerization as well as their medical applications have been largely unexploited. Nowadays most of research works on Lightresponsive copolymers are focused on synthesis and properties of new photo-responsive vehicles in vitro scale (14) However, this strategy despite of its interesting aspect steel is in its primary ages in respect of in vivo investigations.

The present study directed toward designing a special kind of micellar aggregate based on amphiphilic linear-dendritic diblock copolymer. Its structure consists of hydrophilic polyethylene oxide monomethyl ether (PEOM) as hydrophilic block and carbosiloxane denditic branches as hydrophobic block. The dendritic block could be grafted by Magneson II, 4-(4-nitrophenylazo) 1-naphthol, as azo-chromophore which increases hydrophobicity for an amphiphilic character (Figure 1). We used the dendritic structures in the formation of the aggregates since they cause more strong interactions among the chromophores because of its more regular and open arrangement. In this field, recently, we have reported different photoresponsive dendritic and polymeric structures with targeted delivery goals (15-22). As it is known the hydrolytic textural behavior of siliconbased systems is important especially in the area of their applications. Biodegradability in the carbosiloxane linkages are as result of their potentially hydrolysable Si-O linkages. In our previous research, we reported the hydrolytic behavior of this compound as amphiphilic lineardendritic triblock ABA type copolymers based on carbosiloaxe dendrimers (23). Also biocompatibility in the silicon-based dendritic structures as well as silicone-based delivery systems has remarkably developed (24).

Micellar solution Micellar particle
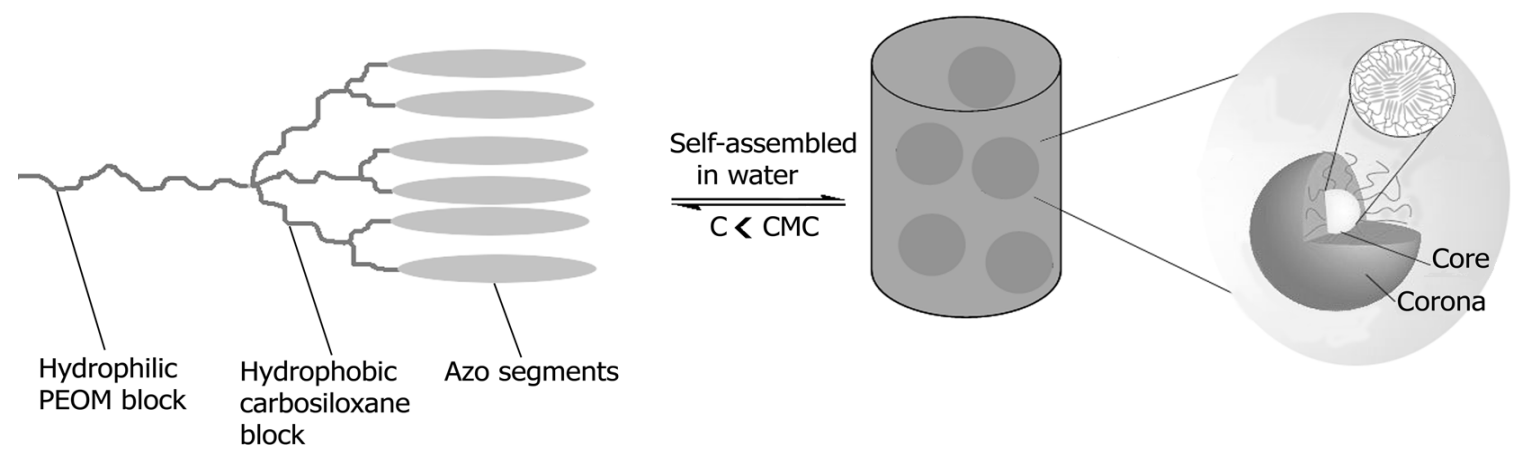

Figure 1. A schematic abstract of amphiphilic diblock copolymer in aqueous medium. 
In this study, for the first time, we report our observations on this particular kind of photoresponsive amphiphilic macromolecules as drug carrier based on first and third steps of a controlled delivery system as explained in the first paragraph. An anti-cancer drug (erlotinib) was selected as bioactive model for incorporation into the carriers.

\section{EXPERIMENTAL}

\section{MATERIALS AND METHODS}

All glasswares were thoroughly dried with vacuum and all reactions were carried out under dried argon atmosphere using standard Schlenk techniques. Karstedt catalyst (Aldrich; platinumdivinyltetramethyldisiloxane complex) was used as received. PEOM (Merck; molecular weight 2000) was precipitated from methylene chloride into n-hexane and dried in vacuum. Allyl bromide and allyl alcohol (Merck) were vacuum-distilled over calcium chloride and both of them were stored on molecular sieves $4 \AA$. TMEDA (Merck; tetramethyl ethylene diamine,) was refluxed with $\mathrm{KOH}$ and freshly distilled. Methyldichlorosilane (Aldrich), trichlorosilane, chlorodimethylsilane, pyrene, benzophenone, and Magneson II (all from Merck) were used without further purification. Erlotinib, was a gift from Drug Applied Research Center (DARC). Dialysis tube, benzoylated (Sigma-Aldrich; Mw cut-off $=1.2 \mathrm{KDa}$ ). Sodium hydride (Merck; $80 \%$ in mineral oil). All solvents were purified by a procedure described in the literature (Schlenk-type solvents and materials) (25).

${ }^{1} \mathrm{H}$ and ${ }^{13} \mathrm{C}$ NMR spectra were obtained on a Bruker AC 300 and $400 \mathrm{MHz}$ spectrometer. Gel Permeation Chromatography (GPC), molecular weights and molecular weight distributions were determined with a Gel Permeation Chromatograph (GPC, WATERS-150 C) equipped with a 410 RI detector, a 510 HPLC pump and m-Styragel columns with pore sizes of 102,103 , and $104 \AA$. The eluent was THF and the molecular weights were calibrated with polystyrene standards. The hydrodynamic diameter of micellar particles was determined with a He-Ne Laser Dynamic Light Scattering (DLS) of Photon Correlation Spectroscopy-SEMATECH. A scattering angle of $90^{\circ}$ was used for the DLS evaluations at $\mathrm{k}=632.8 \mathrm{~nm}$ by cumulant method. Prior to size determination, all the dispersed media were filtered to remove dust particles (25-50 micron, C porosity, Ace Glass Inc., Vineland, N. J.). Results corresponded to the average of three determinations. After vortexing, the hydrodynamic diameter of the micellar particles was determined by DLS measurements before and after UV irradiation. Transmission Electron Microscopy (TEM) experiments were carried out on a LEO 906 Instrument operating at an acceleration voltage of 100 KV. TEM sample was prepared by dipping a copper grid with Formvar film into the freshly prepared particles solution $(0.5 \mathrm{mg} / \mathrm{mL})$. Scanning Electron Microscopy (SEM) was used by LEO 440i conventional microscope under vacuum at an operating voltage of $10 \mathrm{kV}$. Dried sample was gold coated by sputtering for $15 \mathrm{~S}$. UV-Vis spectra were measured over different irradiation time intervals by a SHIMADZU UV-1700 Pharmaspec double-beam Spectrophotometer $\left(10 \mathrm{~mm}\right.$ cell, $\left.\mathrm{H}_{2} \mathrm{O}\right)$. Irradiation was applied by UV mercuric lamp, $366 \mathrm{~nm}$ of (CAMAG, Muttenz-Switzerland) in aqueous media and UV-Vis spectra were recorded over different time intervals. For measuring the thermal cis-trans back isomerization, the samples were kept in a dark oven with constant temperature at $37^{\circ} \mathrm{C}$. Steady-state fluorescence measurements were carried out using a (Shimadzu, Japan) RF-540 spectrofluorometer at $25^{\circ} \mathrm{C}$. Pyrene and benzophenone were used as the fluorescence probe and quencher, respectively. The emission spectra of pyrene was recorded from 350 to $450 \mathrm{~nm}$ after excitation at $335 \mathrm{~nm}$ with the slit widths fixed at 2.5 and $10.0 \mathrm{~nm}$ for the emission and the excitation, respectively.

\section{Synthesis}

Preparation of $\mathrm{CH}_{3}$-PEO-allyl (PEOM-All, 1)

A modified literature procedure was employed (26). Briefly, PEOM-All, 1 was prepared through reaction between PEOM and sodium hydride in THF at $5^{\circ} \mathrm{C}$ (Caution: otherwise the solution produces bubbles and starts to rise). After the evolution of $\mathrm{H}_{2}$ ceased, a solution of allyl bromide in THF was added to the reaction mixture. The crude product was filtered through sintered glass filter and purified by repeated precipitation from methylene chloride into cold nhexane obtaining a white powder, Yeild $66 \% .{ }^{1} \mathrm{H}$ NMR (300 MHz, $\left.\mathrm{CDCl}_{3}\right): \delta 3.33\left(\mathrm{H}_{3} \mathrm{C}, \mathrm{s}, 3 \mathrm{H}\right), 3.48-$ 3.84 (PEOM, m, 180H), $3.98\left(\mathrm{CH}_{2}, \mathrm{~d}, 2 \mathrm{H}\right), 5.11-5.26$ $\left(=\mathrm{CH}_{2}, \mathrm{~m}, 2 \mathrm{H}\right), 5.71-5.93(\mathrm{HC}=, \mathrm{m}, 1 \mathrm{H}) .{ }^{13} \mathrm{C} \mathrm{NMR}$ (300 MHz, $\left.\mathrm{CDCl}_{3}\right): \delta 58.92\left(\mathrm{CH}_{3}\right), 69.39\left(\mathrm{CH}_{2}\right.$, allylic $)$ 70.54 (PEOM), $117.04\left(=\mathrm{CH}_{2}\right), 134.75(\mathrm{CH}=)$. 


\section{General procedure for the preparation of linear- dendritic diblock copolymers}

Here, synthesis of diblock copolymer based on PEOM containing three allyloxy groups (PEOM-All, 3) has been reported as a typical example. The same synthetic method was applied to afford the polymer containing two allyloxy (PEOM-All, 2) groups by using methydichlorosilane as branching agent. A modified literature procedure was employed (25). The operations were carried out in an inert argon atmosphere. Karstedt catalyst $\left(12.6 \times 10^{-5} \mathrm{mmol}, 0.050\right.$ $\mathrm{mL})$ was added to a solution of PEOM-allyl $(0.408 \mathrm{~g}$, $0.200 \mathrm{mmol})$, trichlorosilane $(0.270 \mathrm{~g}, 2 \mathrm{mmol})$ and anhydrous THF $(15 \mathrm{~mL})$. The mixture was kept at $20^{\circ} \mathrm{C}$ for $1 \mathrm{~h}$ under vigorous magnetic stirring and additionally at $40^{\circ} \mathrm{C}$ until no allyl groups were detected by FT IR (1635 $\mathrm{cm}^{-1}$ typically $\left.17 \mathrm{~h}\right)$. Excess trichlorosilane and THF were removed under reduced pressure at $22^{\circ} \mathrm{C} / 3 \mathrm{mmHg}$. Accordingly, a compound containing $\mathrm{Si}-\mathrm{Cl}$ terminal bonds was obtained as a colorless liquid. Then allyl alcohol $(0.069 \mathrm{~g}, 0.001$ mmol) in toluene $(5 \mathrm{~mL})$ was slowly added to a mixture of recently obtained compound $(0.435 \mathrm{~g}$, $0.200 \mathrm{mmol})$ and TMEDA $(0.697 \mathrm{~g}, 0.600 \mathrm{mmol})$ in toluene $(15 \mathrm{~mL})$ at a rate of 30 drops per minute. When addition was finished, the reaction mixture was warmed to $27^{\circ} \mathrm{C}$ for $5 \mathrm{~h}$ and additional 30 minutes at $50^{\circ} \mathrm{C}$. When the reaction was completed by ${ }^{1} \mathrm{H}$ NMR, the salt was filtered off and washed with pentane. The volatile components were removed under reduced pressure, leaving a colorless liquid. The product was purified by repeated precipitation from THF into cold n-hexane and further purification was carried out by column chromatography on silica gel with methanol/chloroform (5/95, v/v) as eluent. After evaporation of the solvents, leaving $0.318 \mathrm{~g}(0.143$ mmol, 71\%), the obtained pure product was a very light yellow waxy matter. ${ }^{1} \mathrm{H}$ NMR $(400 \mathrm{MHz}$, $\left.\mathrm{CDCl}_{3}\right): \delta 0.50-0.57\left(\mathrm{CH}_{2}-\mathrm{Si}, \mathrm{m}, 2 \mathrm{H}\right), 1.60-1.66\left(\mathrm{CH}_{2}\right.$, $\mathrm{m}, 2 \mathrm{H}), 3.35\left(\mathrm{H}_{3} \mathrm{C}, \mathrm{s}, 3 \mathrm{H}\right), 3.53\left(\mathrm{O}-\mathrm{CH}_{2}, \mathrm{~m}, 2 \mathrm{H}\right)$, 3.61-3.63 (PEOM, m, 180H), $3.97\left(\mathrm{CH}_{2}\right.$, allylic, d, 6H), 5.20-5.24 $\left(\mathrm{H}_{2} \mathrm{C}=, \mathrm{m}, 6 \mathrm{H}\right)$. 5.65-5.85 $(\mathrm{CH}=, \mathrm{m}, 3 \mathrm{H})$. ${ }^{13} \mathrm{C}$ NMR $\left(400 \mathrm{MHz}, \mathrm{CDCl}_{3}\right): \delta 6.37\left(\mathrm{CH}_{2}-\mathrm{Si}\right), 23.63$ $\left(\mathrm{CH}_{2}\right), 59.83\left(\mathrm{CH}_{3}\right), 62.94\left(\mathrm{CH}_{2}\right.$, allylic $), 69.52$ (PEO), $72.31\left(\mathrm{O}-\mathrm{CH}_{2}\right), 114.18\left(=\mathrm{CH}_{2}\right), 136.29(\mathrm{CH}=)$.

\section{General procedure for preparation of linear- dendritic diblock copolymers grafted by 4-(4- nitrophenylazo) 1-naphthol}

Here only a typical example of the procedure for the preparation of PEOM-Azo, 6 is shown. The synthetic procedure of PEOM-Azo, 2 was similar to that of PEOM-Azo, 6 except that the reaction was progressed by using chlorodimethylsilane as branching agent. The operations were carried out by using PEOM-All, 3 and the Magneson II in a flask equipped with an argon atmosphere and magnetic stirrer. For this goal, karstedt catalyst $\left(12.60 \times 10^{-5} \mathrm{mmol}, 0.05 \mathrm{~mL}\right)$ was added to a solution of PEOM-All, $3(0.224 \mathrm{~g}, 1$ mmol), methydichlorosilane $(0.345 \mathrm{~g}, 3 \mathrm{mmol})$ and anhydrous THF $(10 \mathrm{~mL})$. The mixture was kept at $20^{\circ} \mathrm{C}$ for $1 \mathrm{~h}$ under vigorous magnetic stirring and additionally at $40^{\circ} \mathrm{C}$ until no allyl groups were detected by FT IR $\left(1635 \mathrm{~cm}^{-1}\right.$ typically $\left.17 \mathrm{~h}\right)$. Excess methydichlorosilane and THF were removed under reduced pressure at $22^{\circ} \mathrm{C} / 3 \mathrm{mmHg}$. The obtained compound containing $\mathrm{Si}-\mathrm{Cl}$ terminal bands, a very light yellow liquid, immediately was exposed to reaction of the next step. Thus, Magneson II (0.176 g, $0.600 \mathrm{mmol})$ in chloroform $(30 \mathrm{~mL})$ was slowly added to a mixture of recently obtained compound $(0.258 \mathrm{~g}, 0.100 \mathrm{mmol})$ and TMEDA $(0.035 \mathrm{~g}, 0.300$ mmol) in chloroform $(5 \mathrm{~mL})$ at a rate of 30 drops per minute. When addition was finished the reaction mixture was warmed to $27^{\circ} \mathrm{C}$ for $15 \mathrm{~h}$ and additional 30 minutes at $50^{\circ} \mathrm{C}$. When the reaction was completed by ${ }^{1} \mathrm{H}$ NMR, the salt was filtered off and washed with pentane. The volatile components were removed under reduced pressure, leaving a red liquid. After filtration, the product was purified by repeated precipitation from THF and chloroform into cold nhexane and further purification was carried out by exhaustive dialysis bag (molecular cut-off $=1.2 \mathrm{kDa}$ ) for $72 \mathrm{~h}$ with THF change at $6 \mathrm{~h}$ intervals. PEOMAzo, 6 was obtained as light red matter after evaporation of the solvents, leaving $0.218 \mathrm{~g}(0.053$ mmol, 53\%). ${ }^{1} \mathrm{H}$ NMR (400 $\left.\mathrm{MHz}, \mathrm{CDCl}_{3}\right): \delta 0.06$ $\left(\mathrm{H}_{3} \mathrm{C}-\mathrm{Si}, \mathrm{s}, 9 \mathrm{H}\right), \quad 0.80-0.93\left(\mathrm{CH}_{2}-\mathrm{Si}, \mathrm{m}, 8 \mathrm{H}\right), 1.20-$ $1.35\left(\mathrm{CH}_{2}, \mathrm{~m}, 8 \mathrm{H}\right), 3.41\left(\mathrm{H}_{3} \mathrm{C}, \mathrm{s}, 3 \mathrm{H}\right), 3.53\left(\mathrm{O}-\mathrm{CH}_{2}\right.$, $\mathrm{m}, 8 \mathrm{H}), 3.60-3.63$ (PEOM, m, 180H), 6.97-8.38 $\left(\mathrm{CH}_{\text {aromatic }}, 60 \mathrm{H}\right) .{ }^{13} \mathrm{C}$ NMR $\left(400 \mathrm{MHz}, \mathrm{CDCl}_{3}\right): \delta-$ $1.02\left(\mathrm{Si}-\mathrm{CH}_{3}\right), 8.10\left(\mathrm{CH}_{2}-\mathrm{Si}\right), 22.18\left(\mathrm{CH}_{2}\right), 60.70(\mathrm{Si}-$ $\left.\mathrm{O}-\mathrm{CH}_{2}\right), 69.54$ (PEO), $72.59\left(\mathrm{O}-\mathrm{CH}_{2}\right), 115.11$, $123.37,124.74,126.21,126.68,126.84,126.95$, $133.00,133.75,134.27,136.33,138.75,143.33$ and $146.35\left(\mathrm{C}_{\text {aromatic }}\right.$ and $\left.\mathrm{CH}_{\text {aromatic }}\right)$.

General procedure to prepare of PEOM-Azo, 2 and PEOM-Azo, 6 micellar solutions

The solutions were prepared by a reported literature procedure (25). Briefly, the PEOM-Azo, 2 and PEOM-Azo, 6 systems were dissolved in a few drops 
of THF. Then it was added to redistilled water at the rate of 1 drop every 10 seconds under vigorous stirring. Subsequently, the THF in the aqueous solution was removed in an open air during the agitation. The remained micelle solution was diluted with redistilled water.

\section{Photoisomerization of PEOM-Azo, 2 and PEOM- Azo, 6 systems}

Photoisomerization of the systems $(2 \mathrm{mg} / \mathrm{ml})$ were investigated using irradiation at $366 \mathrm{~nm}$ in aqueous solution. The absorbance at $411 \mathrm{~nm}$ which corresponds to the $\pi-\pi^{*}$ transition, trans azo-group, decreased with time and the photostationary state was reached within $35 \mathrm{~min}$ of UV irradiation. The solutions were then kept in the dark and absorbance was recorded at different time intervals to study the back-isomerization process.

\section{Determination of CMC}

CMC was determined by UV-Vis spectrophotometer. Pyrene-saturated PEOM-Azo, 2 and PEOM-Azo, 6 solutions were prepared by adding excessive pyrene into different concentrations of PEOM-Azo, 2 and PEOM-Azo, 6 solutions. At first, the PEOM-Azo, 2 and PEOM-Azo, 6 and pyrene dissolved in THF then were added dropwise into water under magnetic stirring. In order to make sure the completely solubilization of pyrene by PEOM-Azo, 2 and PEOM-Azo, 6 systems, the resultant aqueous dispersion were sonicated for $30 \mathrm{~min}$. After keeping at room temperature for 3 hours, small amounts of precipitates were removed by centrifugation.

\section{Determination of aggregation number}

Steady-state fluorescence measurements were carried out according to reported procedure (27). The aggregation number of micelle were determined using pyrene as the luminescent probe in a concentration of $10^{-6} \mathrm{~mol} . \mathrm{L}^{-1}$, and benzophenone as a quencher in a concentration range of $\left(0-0.1 \times 10^{-5} \mathrm{~mol} . \mathrm{L}^{-1}\right)$. The concentration of each micellar solutions was less than five times the CMC to avoid the probable formation of polydispersion micellar aggregates, which would cause inaccuracies in determination of aggregation number (28). The solutions were sonicated for 15 min. After standing at room temperature for 3 hours, they were applied to spectroscopic measurements.

\section{Drug-incorporation}

Drug-incorporation into PEOM-Azo, 2 and PEOMAzo, 6 carriers in trans form

In this section the drug:carrier ratio was varied from $0.1: 1.0$ to $0.8: 1.0$ (Table 3) for the preparation of drug-loaded systems. The PEOM-Azo, 2 and PEOMAzo, 6 carriers were dissolved in $10 \mathrm{~mL}$ THF and these solutions were added to a round-bottom flask equipped with reflux condenser and magnetic stirrer containing a solution of hydrophobic (erlotinib) and hydrophilic (erlotinib hydrochloride) drugs in $10 \mathrm{~mL}$ THF. The solutions were stirred for $2 \mathrm{~h}$ at ambient temperature. To remove free drug, the solution was purified by microfiltration and then precipitated in cold n-hexane. The drug-incorporated carriers were dried in vacuum at room temperature to yield white (erlotinib hydrochloride) and yellow (erlotinib) compounds; the respective data for Incorporation Content (IC) and drug Loading Efficiency (LE) of PEOM-Azo, 2 and PEOM-Azo, 6 carriers are determined (29) based on equations (1) and (2) ( Table 3).

$$
\begin{aligned}
& \mathrm{IC}(\mathrm{wt} \%)=100 \times \frac{\text { the total amountof drugin carrier }}{\text { the amountof carrieraddedinitially }} \\
& \mathrm{LE}(\%)=100 \times \frac{\text { the totalamountof drugin carrier }}{\text { theamountof drugaddedinitially }}
\end{aligned}
$$

The amount of trapped drug into PEOM-Azo, 2 and PEOM-Azo, 6 carriers was measured with UV-Vis spectrophotometer as well as, in second procedure, the weights of carrier were determined before and after incorporation; their differences are the amount of trapped drug in the carriers.

\section{Drug-incorporation into PEOM-Azo, 2 and PEOM-Azo, 6 carriers in cis form}

The procedure was similar to method applied to the carriers in trans form, except that prior to incorporating the hydrophobic and hydrophilic drugs (erlotinib and erlotinib hydrochloride) the solution containing PEOM-Azo, 2 and PEOM-Azo, 6 were placed at the exposure of UV irradiation in order to generate cis forms of PEOM-Azo, 2 and PEOM-Azo, 6 systems. Drug-incorporation process of the carriers in cis form was carried out in the dark room. 
In vitro release kinetics

General procedure to study release of drugs from PEOM-Azo, 2 and PEOM-Azo, 6 carriers

With regard to the highest drug IC at drug:carrier ratio of $0.1: 1.0$ and $0.2: 1.0$, all samples were prepared at the same ratio. The drug-loaded carriers which prepared in the previous section were dissolved into a $0.5 \mathrm{~mL}$ of $\mathrm{THF}$, as a volatile solvent, and the resulting solutions were poured gently into $3 \mathrm{~mL}$ aqueous phosphate buffered solution at $\mathrm{pH} 7.4$ under vigorous agitation. The organic solvent was removed in an open air during the agitation. The bulk solution was stirred by a magnetic stirrer continuously at $800 \mathrm{rev} / \mathrm{min}$ and a sample solution $(3 \mathrm{~mL})$ was withdrawn at regular selected intervals and replaced with equal volumes of the media. The quantity of released drug was analyzed by means of UV-Vis spectrophotometer and determined from the calibration curve obtained previously under the same conditions. Studies of the release kinetics of drug-incorporated carriers in cis form were carried out in the dark room.

Release of erlotinib from PEOM-Azo, 2 and PEOM-Azo, 6 systems under the UV irradiation

In order to investigate the effect of UV irradiation during the release process of PEOM-Azo [2, 6], a set of two experiments was carried out by drug:carrier ratio of $0.1: 1.0$. One of the solutions containing erlotinib-incorporated PEOM-Azo, 2 and PEOM-Azo, 6 carriers in trans form was kept outside while the second one was placed inside the UV irradiation. Both experiments continued according to the general procedure to investigate release kinetics.

\section{Statistical data treatment}

All experiments were performed at least in triplicate and the results averaged. Error bars in the figures and error values given in the tables are the calculated standard deviations.

\section{RESULTS}

\section{Characterization}

The diblock copolymers synthesized in this work have $\mathrm{AB}$ type consisted of lineardendritic parts. The completion of hydrosilylation reaction was monitored by FT IR spectroscopy during the reaction, which confirmed the consumption of peripheral allyloxy groups until no allyl peak were detected by FT IR $\left(1635 \mathrm{~cm}^{-1}\right)$. Also the synthesis of PEOM-Azo, 2 and PEOM-Azo, 6 was confirmed through ${ }^{1} \mathrm{H}$ NMR spectroscopy which clearly reveals the disappearance of allyloxy protons at regions 5.14, 5.22 and $5.84 \mathrm{ppm}$ and appearance of aromatic porotons between 6.978.38 ppm. Furthermore, GPC experiments clearly exhibit that molecular weights of azo-functionalized macromolecules have narrow distribution, implying formation of regular structures. Figure 2 shows the GPC curves of the PEOM-Azo, 2 and PEOM-Azo, 6 systems.

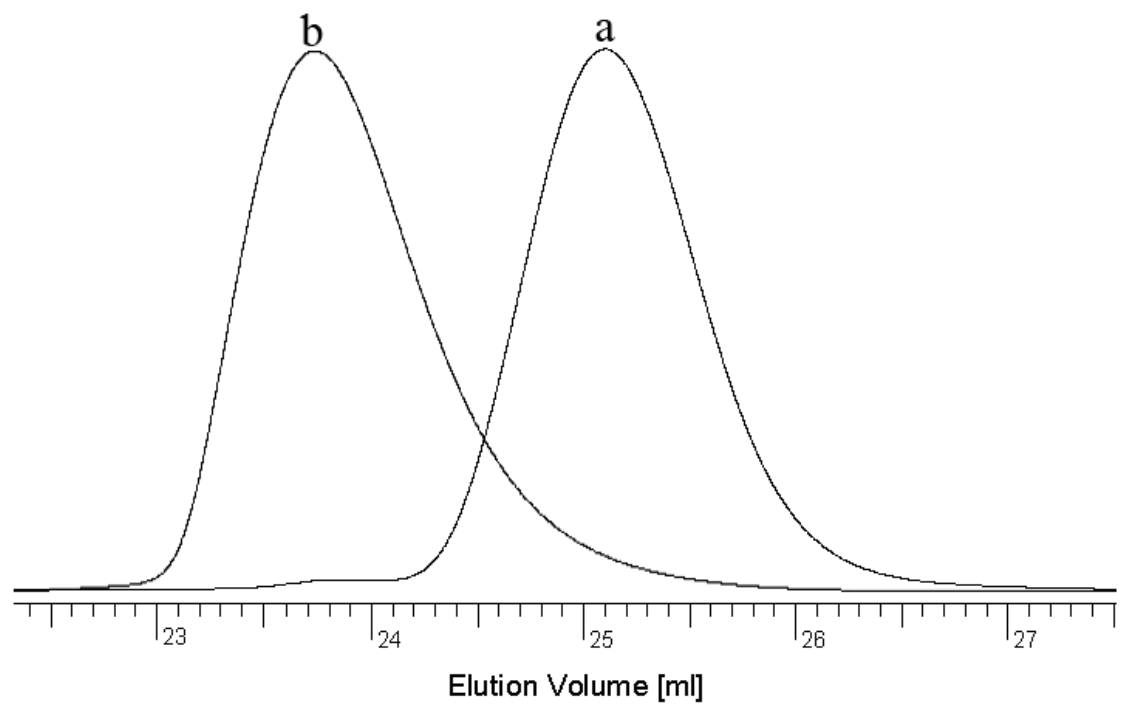

Figure 2. GPC chromatogram of the PEOM-Azo 2 (a) and PEOM-Azo 6 (b). 
Table 1. Physicochemical Properties of the PEOM-Azo, 2 and PEOM-Azo, 6 systems. The characteristics of the PEOM-Azo, 2 and PEOM-Azo, 6 are presented in Table 1.

\begin{tabular}{ccccc}
\hline Generation & $\begin{array}{c}\text { Hydrophobic } \\
\text { Block }\left(\mathrm{X}_{\mathrm{w}}\right)^{\mathrm{a}}\end{array}$ & $\begin{array}{c}\text { PDI } \\
\left(\mathrm{M}_{\mathrm{w}} / \mathrm{M}_{\mathrm{n}}\right)^{\mathrm{b}}\end{array}$ & $\begin{array}{c}\text { PDI } \\
\left(\mu_{2} / \Gamma^{2}\right)^{\mathrm{c}}\end{array}$ & $\begin{array}{c}\mathrm{D} \\
(\mathrm{nm})^{\mathrm{d}}\end{array}$ \\
\hline PEOM-Azo, 2 & 44.89 & 1.051 & 0.049 & $231.7 \pm 1.2$ \\
PEOM-Azo, 6 & 66.39 & 1.043 & 0.039 & $264.4 \pm 1.4$ \\
\hline
\end{tabular}

${ }^{a}$ Calculated for hydrophobic dendritic carbosiloxane blocks functionalized by magneson II as azo chromophore;

${ }^{b}$ Polydispersity index (PDI) measured by GPC; ${ }^{c}$ Polydispersity index (PDI) measured by DLS in water; ${ }^{\mathrm{d}}$ Diameter measured by dynamic light scattering in water, the data represent the mean $\pm \mathrm{SD}$ (standard deviation), $\mathrm{n}=3$.

\section{Photoisomerization of PEOM-Azo, 2 and PEOM-Azo, 6 systems}

Exposure of PEOM-Azo, 2 and PEOM-Azo, 6 to UV light at $366 \mathrm{~nm}$ for $35 \mathrm{~min}$ for inducing sufficient trans-cis photoisomerization led to a noticeable reduction in the trans azo-groups $\left(\pi-\pi^{*}\right)$ absorption band and a simultaneous increase in the cis azo-groups $\left(n-\pi^{*}\right)$ absorption band at $474 \mathrm{~nm}$. Moreover, the position of the $\pi$ $\pi^{*}$ absorption band of PEOM-Azo, 2 and PEOM-Azo, 6 systems showed a small hypsochromic shift during irradiation until a photostationary state is reached.
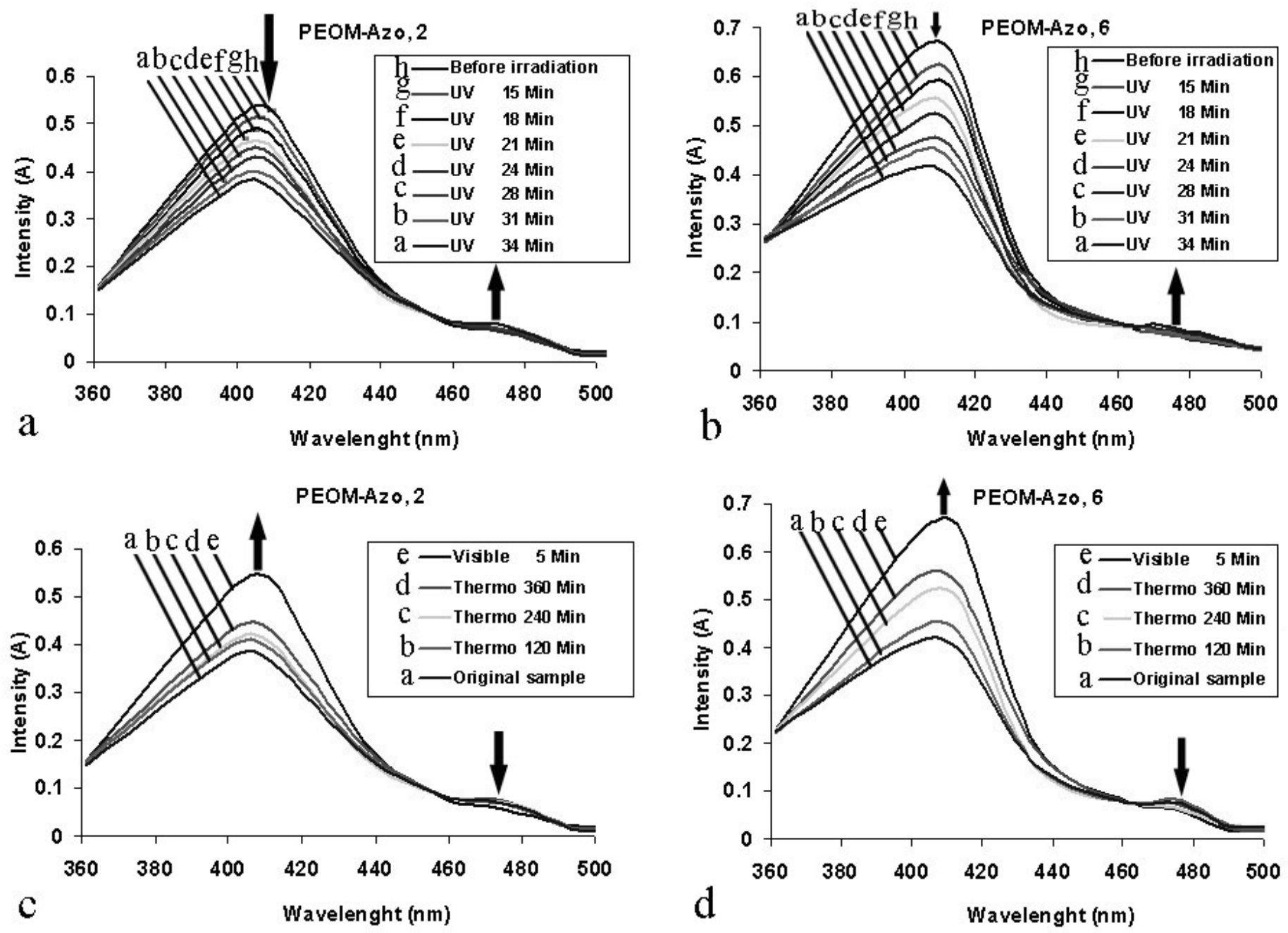

Figure 3. UV/Vis absorption spectra: (a) and (b) after UV irradiation of PEOM-Azo, 2 and PEOM-Azo, 6 systems; (c) and (d) back isomerization showing visible-light and thermal recovery of PEOM-Azo, 2 and PEOM-Azo, 6 systems in aqueous medium at $37^{\circ} \mathrm{C}$. 
The cis-trans back isomerization in the PEOM-Azo, 2 and PEOM-Azo, 6 was studied in order to determine the stability of the cis form within the systems, which is essential for sustained release of drugs. The UV/Vis spectra of the PEOM-Azo, 2 and PEOM-Azo, 6 were obtained in the dark, keeping two isosbestic points at 361 and $464 \mathrm{~nm}$ indicating that conversion between only two species, trans and cis forms of PEOM-Azo, 2 and PEOM-Azo, 6 occurs in solution.

PEOM-Azo, 2 and PEOM-Azo, 6 as micellar aggregates and $\mathrm{CMC}$ determination

The PEOM-Azo, 2 and PEOM-Azo, 6 systems and pyrene were dissolved in THF then added dropwise into water under magnetic agitation. Because of miscibility of THF and water, they mix with each other rapidly as soon as solution mixture of PEOMAzo, 2 and PEOM-Azo, 6 and pyrene are added. After removal of THF, a yellow PEOM-Azo, 2 and PEOMAzo, 6 micellar solution was formed. Pyrene's solubility in water is only $7.2 \times 10^{-4} \mathrm{~mol} / 1(336 \mathrm{~nm}$ as maximum absorption wavelength). Thus, by determining the amount of pyrene solubilized in aqueous solution, CMC value of PEOM-Azo, 2 and PEOM-Azo, 6 micelles could be determined.

Determination of micellar aggregation numbers The aggregation number $\left(\mathrm{N}_{\mathrm{agg}}\right)$ of the monomeric particles in the micellar aggregates can be determined by a fluorescence quenching technique (27). This method is based on quenching of pyrene fluorescence by a hydrophobic quencher. Benzophenone was used as the quencher. By changing the quencher concentration $\mathrm{C}_{\mathrm{q}}$, the corresponding equation (3) is as follows:

$\operatorname{Ln}\left(\mathbf{I}_{0} / \mathbf{I}\right)=\left[\mathbf{N}_{\text {agg }} /\left(\mathbf{C}_{\text {mic }}-\mathbf{C}_{\text {CMC }}\right)\right] \times \mathbf{C}_{\mathbf{q}}$

Where $\mathrm{I}_{0}$ and $\mathrm{I}$ are the fluorescence intensities of pyrene in the absence and presence of the quencher;
$\mathrm{C}_{\mathrm{q}}$ and $\mathrm{C}_{\text {mic }}$ are the molar concentration of the quencher and PEOM-Azo, 2 and PEOM-Azo, 6 systems, respectively. The relevant $\mathrm{N}_{\text {agg }}$ values were obtained from the linear relationship by applying equation, and they are listed in Table 2.

\section{Size and size distribution of the micellar} aggregates

Figures $6 \mathrm{a}$ and $6 \mathrm{~b}$ show typical TEM and SEM micrographs of PEOM-Azo, 6 aggregates in trans and cis form, respectively. TEM observations of cis form could not be experimented due to the limitation of the techniques available to us. However, PEOM-Azo, 2 and PEOM-Azo, 6 and their drug-incorporated carriers were studied by DLS to determine the effect of UV irradiation on the size of the aggregates (Table 3 ).

\section{Drug-Incorporation Content (IC) and Loading Efficiency (LE)}

The IC and LE of the drugs in PEOM-Azo, 2 and PEOM-Azo, 6 systems were analyzed in different drug:carrier ratio by weight in trans and cis form of the carriers; the obtained results are shown in Table 4.

\section{In vitro release kinetics}

In order to evaluate hydrolytic behavior of PEOMAzo, 2 and PEOM-Azo, 6 systems as drug carriers, some experiments were carried out in different conditions and the obtained results are shown in Figure 7.

\section{DISCUSSION}

\section{Reaction conditions}

Self-assembling of amphiphilic copolymers in an aqueous media lead to forming of polymeric micellar aggregates.

Table 2. Micellar Properties of the PEOM-Azo, 2 and PEOM-Azo, 6 systems.

The CMC values so were calculated for PEOM-Azo, 2 and PEOM-Azo, 6 were reported in Table 2.

\begin{tabular}{rcccccc}
\hline Micelles & \multicolumn{2}{c}{$\mathrm{CMC}(\mathrm{mg} / \mathrm{L})$} & \multicolumn{3}{c}{$\mathrm{N}_{\mathrm{agg}}$} & \multicolumn{2}{c}{ T Total numbers of azo-groups } \\
\cline { 2 - 7 } & $\mathrm{E}$ & $\mathrm{Z}$ & $\mathrm{E}$ & $\mathrm{Z}$ & $\mathrm{E}$ & $\mathrm{Z}$ \\
\hline PEOM-Azo, 2 & 10.7 & 18.6 & 32 & 78 & 64 & 156 \\
PEOM-Azo, 6 & 7.1 & 14.7 & 9 & 33 & 54 & 198 \\
\hline
\end{tabular}



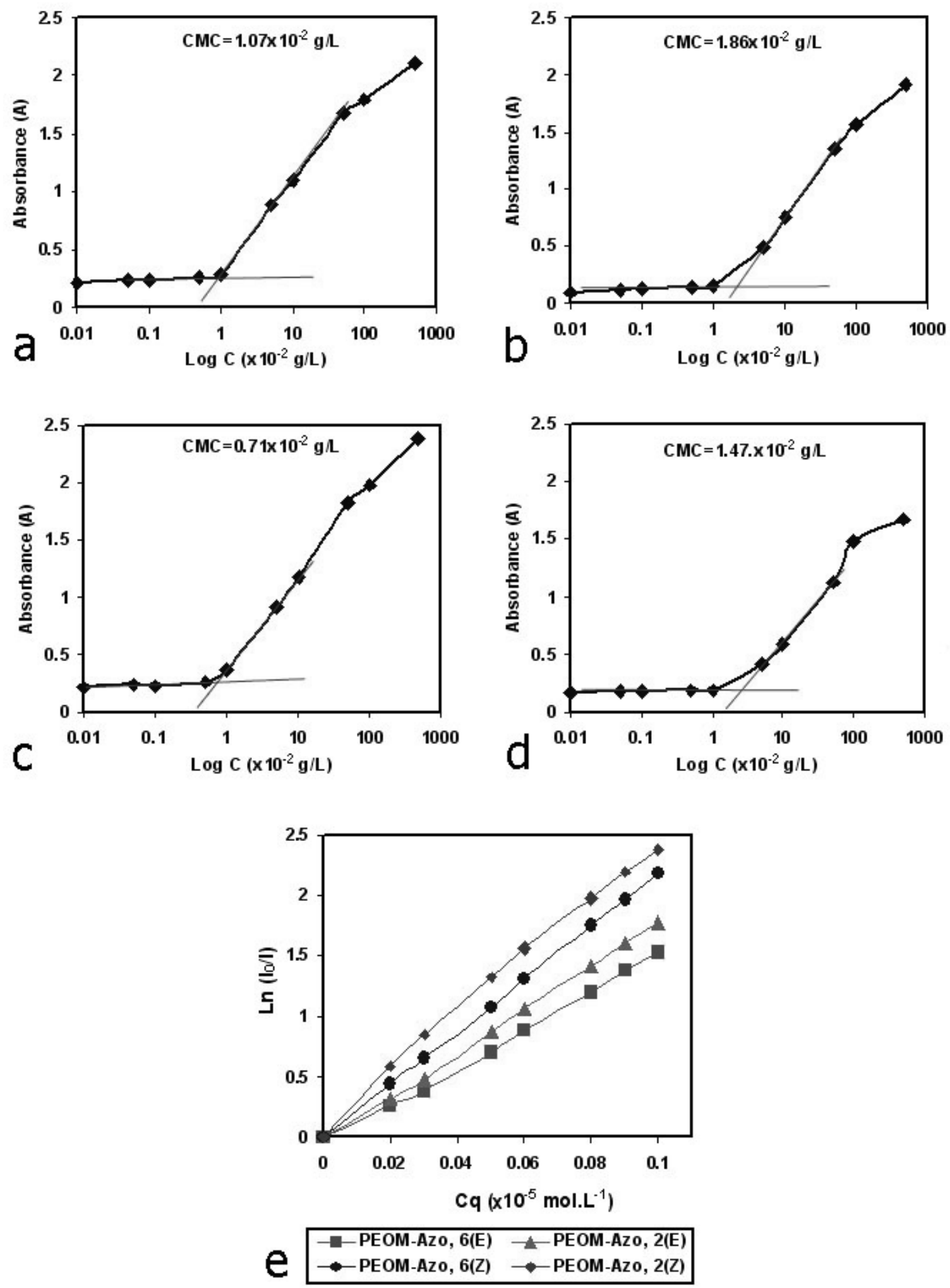

Figure 5. Absorbance of pyrene at $335 \mathrm{~nm}$ in aqueous solution at $25^{\circ} \mathrm{C}$ : (a) PEOM-Azo, 2 (E); (b) PEOM-Azo, 2 (Z); (c) PEOM-Azo, 6 (E); (d) PEOM-Azo, 6 (Z); (e) $\ln \left(\mathrm{I}_{0} / \mathrm{I}\right)$ of pyrene as a function of concentration of the quencher, benzophenone, in PEOM-Azo, 2 and PEOM-Azo, 6 aqueous solution at $25^{\circ} \mathrm{C}$. 


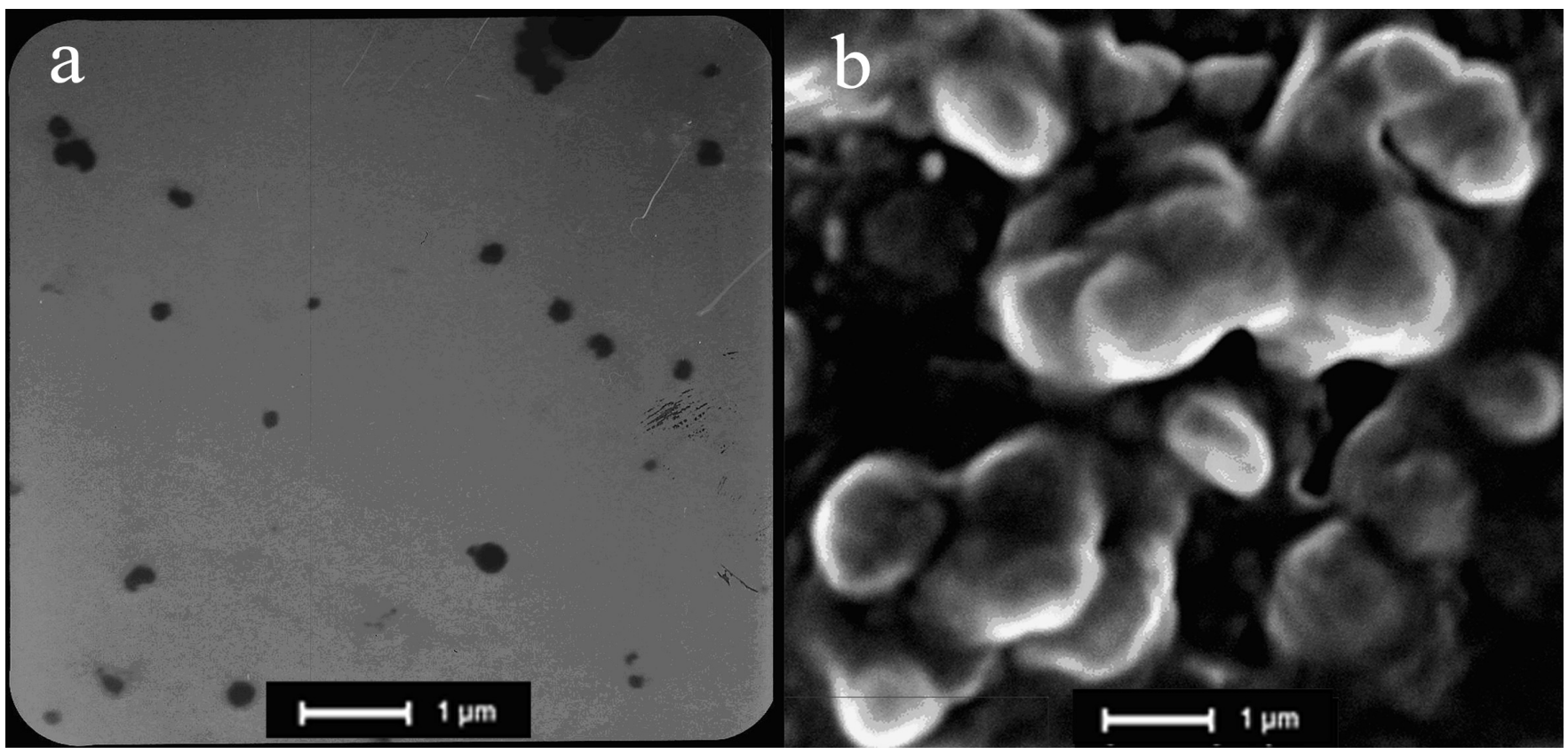

Figure 6. Morphology of PEOM-Azo, 6 aggregates incorporated by erlotinib observed by (a) TEM in drug:carrier ratio (by weight) of 0.1:1.0 in trans form and (b) SEM in drug:carrier ratio (by weight) of 0.2:1.0 in cis form.

Table 3. Characteristic data of the various PEOM-Azo, 2 and PEOM-Azo, 6 carriers, measured by DLS. The average sizes of the drug-incorporated and the unloaded carriers, measured by DLS, were displayed in Table 3.

\begin{tabular}{ccccc}
\hline Carriers & \multicolumn{2}{c}{$\mathrm{D}(\mathrm{nm})^{\mathrm{c}}$} & $\mathrm{PDI}^{\mathrm{c}}\left(\mu_{2} / \Gamma^{2}\right)^{\mathrm{f}}$ \\
\hline & $\mathrm{E}$ & $\mathrm{Z}$ & $\mathrm{E}$ & $\mathrm{Z}$ \\
PEOM-Azo, 2 & $231.7 \pm 1.4$ & $738.0 \pm 0.6$ & 0.049 & 0.055 \\
PEOM-Azo, 6 & $264.4 \pm 0.3$ & $937.8 \pm 0.9$ & 0.039 & 0.086 \\
PEOM-Azo, 2 & $250.8^{\mathrm{d}} \pm 1.6$ & $877.6^{\mathrm{e}} \pm 1.5$ & 0.098 & 0.014 \\
PEOM-Azo, 6 $^{\mathrm{a}}$ & $328.7^{\mathrm{d}} \pm 0.4$ & $1314.9^{\mathrm{e}} \pm 1.3$ & 0.017 & 0.011 \\
PEOM-Azo, 2 & $238.0^{\mathrm{d}} \pm 1.8$ & $985.1^{\mathrm{e}} \pm 0.9$ & 0.010 & 0.032 \\
PEOM-Azo, 6 & $285.3^{\mathrm{d}} \pm 0.1$ & $1314.9^{\mathrm{e}} \pm 1.2$ & 0.052 & 0.046 \\
\hline
\end{tabular}

${ }^{a}$ Incorporated by erlotinib; ${ }^{b}$ Incorporated by erlotinib hydrochloride; ${ }^{c}$ Diameter measured by dynamic light scattering in water, the data represent the mean $\pm \mathrm{SD}$ (standard deviation), $\mathrm{n}=3$; ${ }^{\mathrm{d}}$ Drug: carrier ratio (by weight) as $0.1: 1.0$;

${ }^{\mathrm{e}}$ Drug:carrier ratio (by weight) as $0.2: 1.0 ;{ }^{\mathrm{f}}$ Polydispersity index (PDI) measured by DLS in water; E: trans form; Z: cis form.

In an effort to obtain systems with ability to act as a drug carrier, a simple amphiphilic diblock copolymer was synthesized based on hydrophilic linear PEOM and hydrophobic dendritic carbosiloxane branches grafted by two and six magneson II moiety as PEOM-Azo, 2 and PEOMAzo, 6. As was depicted in Scheme 1, the synthetic path begins with obtaining PEOM-All, 1 which then underwent repeated hydrosilylation-alcoholysis to build dendritic carbosiloxane branches onto linear
PEOM (25). The allylation of PEOM was achieved by allowing deprotonated-PEOM to react with allyl bromide (25). The peripheral allyloxy groups of linear PEOM were reacted with a derivative of chlorosilanes, as branching reagent, in the presence of Karstedt catalyst. Then, allylalcohol was added as alcoholysis reagent. This cycle was repeated for second time to prepare PEOM-Azo, 2 and PEOMAzo, 6 systems, except that alcoholysis reagent was an azo chromophore. 
Table 4. Effect of drug:carrier ratios on drug IC and LE of PEOM-Azo, 2 and PEOM-Azo, 6 systems.

\begin{tabular}{|c|c|c|c|c|c|}
\hline \multirow[t]{2}{*}{ Carriers } & \multirow[t]{2}{*}{ Drug:carrier ratio (by weight) } & \multicolumn{2}{|c|}{$\mathrm{IC}(\mathrm{LE})_{\mathrm{Erl}}$} & \multicolumn{2}{|c|}{ IC (LE) Erl. hyd. } \\
\hline & & $\mathrm{E}$ & Z & $\mathrm{E}$ & Z \\
\hline \multirow[t]{4}{*}{ PEOM-Azo, 2} & $0.1: 1.0$ & $\begin{array}{c}2.6 \pm 0.7 \\
(26.2 \pm 1.3)\end{array}$ & $\begin{array}{c}4.8 \pm 0.4 \\
(48.3 \pm 0.7)\end{array}$ & $\begin{array}{c}0.6 \pm 0.2 \\
(6.6 \pm 0.7)\end{array}$ & $\begin{array}{c}6.4 \pm 1.1 \\
(64.5 \pm 1.7)\end{array}$ \\
\hline & $0.2: 1.0$ & $\begin{array}{c}2.9 \pm 0.9 \\
14.6 \pm 0.5)\end{array}$ & $\begin{array}{c}10.3 \pm 1.5 \\
(51.7 \pm 1.7)\end{array}$ & $\begin{array}{c}0.7 \pm 0.2 \\
(3.8 \pm 0.3)\end{array}$ & $\begin{array}{c}13.8 \pm 1.4 \\
(69.1 \pm 1.9)\end{array}$ \\
\hline & $0.4: 1.0$ & $\begin{array}{c}0.5 \pm 0.1 \\
(1.4 \pm 0.1)\end{array}$ & $\begin{array}{c}4.9 \pm 0.2 \\
(12.4 \pm 0.6)\end{array}$ & $\begin{array}{c}0.7 \pm 0.1 \\
(1.9 \pm 0.2)\end{array}$ & $\begin{array}{c}7.3 \pm 1.3 \\
(18.3 \pm 1.0)\end{array}$ \\
\hline & 0.8:1.0 & $\begin{array}{c}0.1 \pm 0.0 \\
(0.1 \pm 0.0)\end{array}$ & $\begin{array}{c}0.2 \pm 0.1 \\
(0.2 \pm 0.1)\end{array}$ & $\begin{array}{c}0.2 \pm 0.1 \\
(0.3 \pm 0.1)\end{array}$ & $\begin{array}{c}0.4 \pm 0.1 \\
(0.5 \pm 0.1)\end{array}$ \\
\hline \multirow[t]{4}{*}{ PEOM-Azo, 6} & $0.1: 1.0$ & $\begin{array}{c}5.2 \pm 0.8 \\
(52.4 \pm 2.5)\end{array}$ & $\begin{array}{c}5.9 \pm 1.1 \\
(59.4 \pm 1.7)\end{array}$ & $\begin{array}{c}0.5 \pm 0.2 \\
(5.4 \pm 0.4)\end{array}$ & $\begin{array}{c}7.3 \pm 1.5 \\
(73.1 \pm 1.4)\end{array}$ \\
\hline & $0.2: 1.0$ & $\begin{array}{c}5.6 \pm 0.9 \\
(28.2 \pm 1.7)\end{array}$ & $\begin{array}{c}12.8 \pm 1.0 \\
(64.3 \pm 1.4)\end{array}$ & $\begin{array}{c}0.5 \pm 0.1 \\
(2.6 \pm 0.2)\end{array}$ & $\begin{array}{c}15.3 \pm 1.6 \\
(76.6 \pm 1.8)\end{array}$ \\
\hline & $0.4: 1.0$ & $\begin{array}{c}0.6 \pm 0.2 \\
(1.7 \pm 0.1)\end{array}$ & $\begin{array}{c}7.3 \pm 0.5 \\
(18.4 \pm 0.4)\end{array}$ & $\begin{array}{c}0.7 \pm 0.2 \\
(1.8 \pm 0.1)\end{array}$ & $\begin{array}{c}9.8 \pm 1.3 \\
(24.6 \pm 1.1)\end{array}$ \\
\hline & 0.8:1.0 & $\begin{array}{c}0.3 \pm 0.1 \\
(0.4 \pm 0.1)\end{array}$ & $\begin{array}{c}0.4 \pm 0.1 \\
(0.5 \pm 0.1)\end{array}$ & $\begin{array}{c}0.4 \pm 0.1 \\
(0.6 \pm 0.1)\end{array}$ & $\begin{array}{c}0.6 \pm 0.1 \\
(0.8 \pm 0.1)\end{array}$ \\
\hline
\end{tabular}

IC: Incorporation Content (wt \%); LE: Loading Efficiency (wt \%), the data represent the mean $\pm \mathrm{SD}$ (standard deviation), $(\mathrm{n}=3)$; E: trans form; Z: cis form; Erl.: Erlotinib; erl. hyd.: Erlotinib hydrochloride.
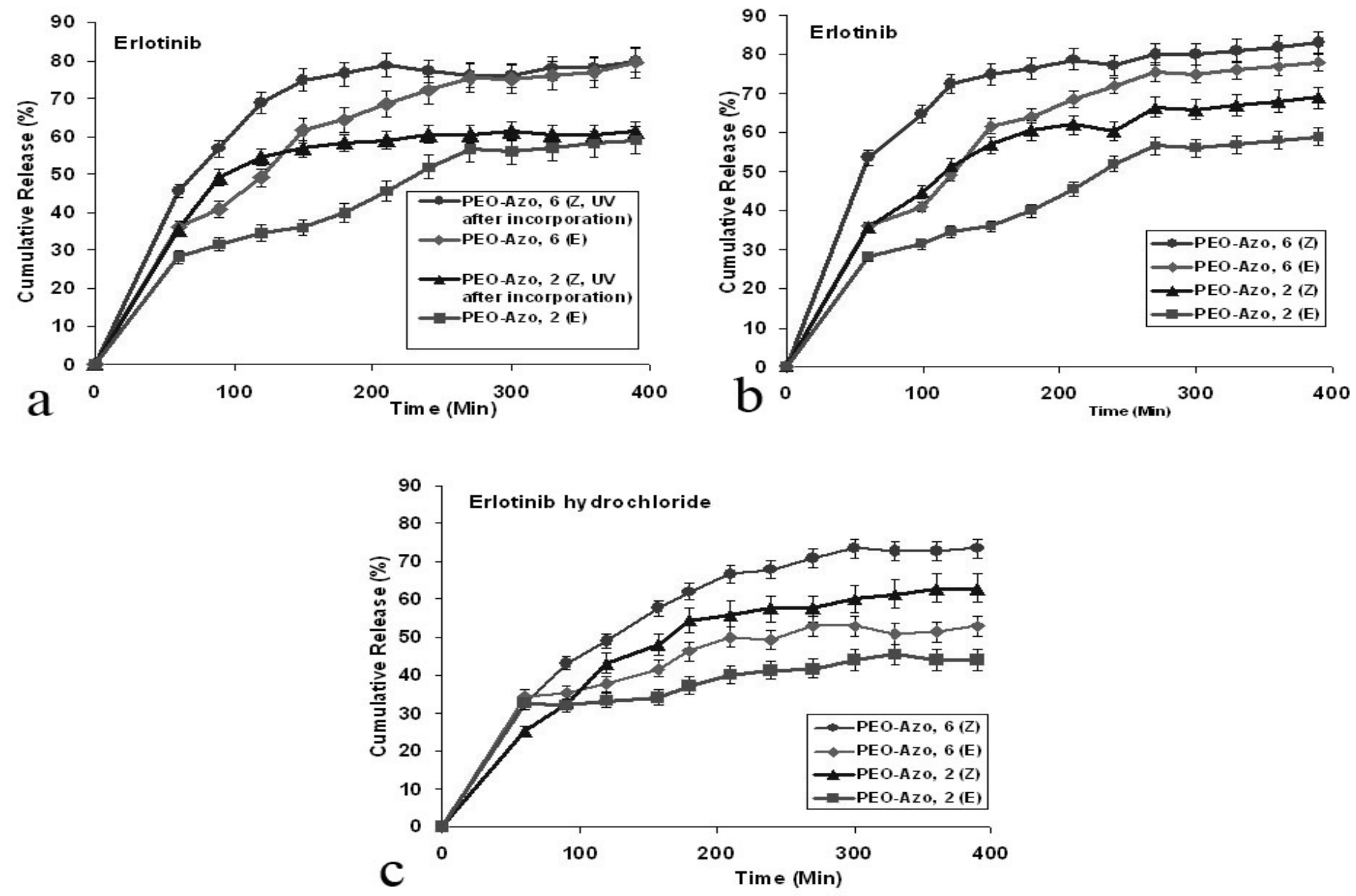

Figure 7. In vitro release of drug as a function of time at $\mathrm{pH} 7.4$; $\mathrm{E}$ : trans form and $\mathrm{Z}$ : cis form: (a) erlotinib during UV irradiation after incorporation from PEOM-Azo, 2 and PEOM-Azo, 6 systems (b) erlotinib from PEOM-Azo, 2 and PEOM Azo, 6 systems (c) erlotinib hydrochloride from PEOM-Azo, 2 and PEOM-Azo, 6 systems. 


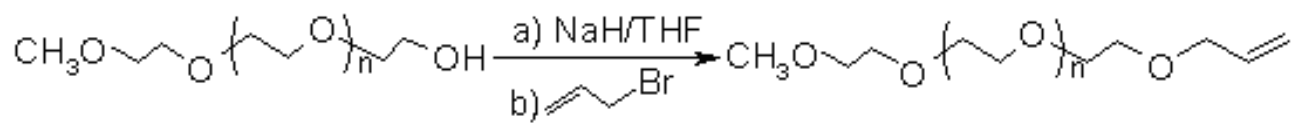

PEOM-AII, 1

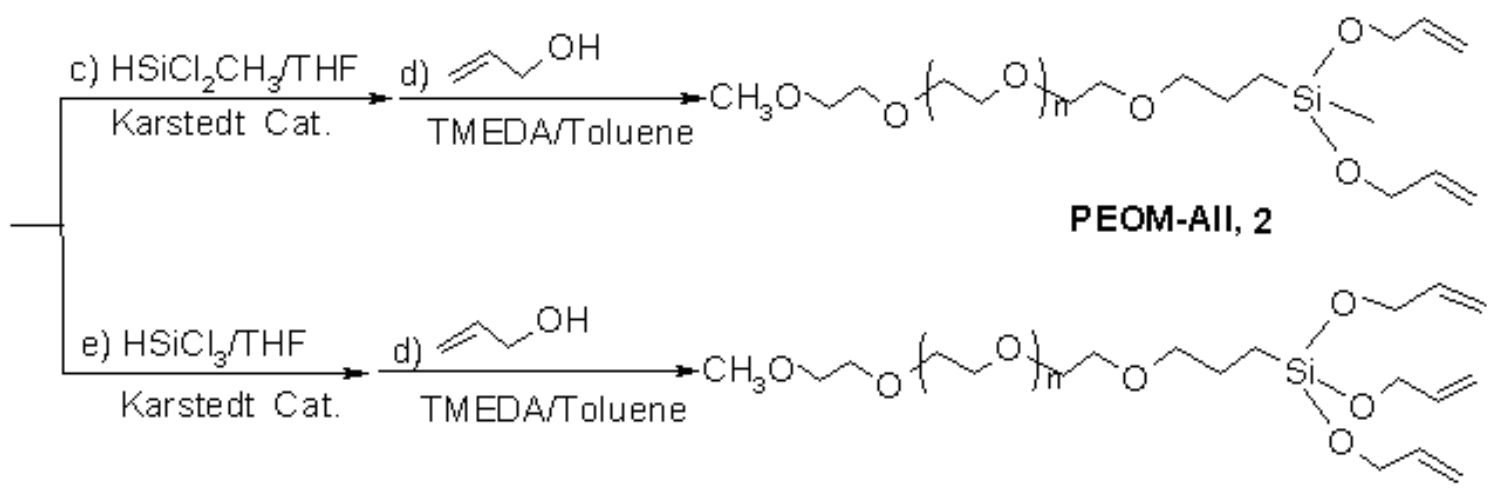

PEOM-AII, 3

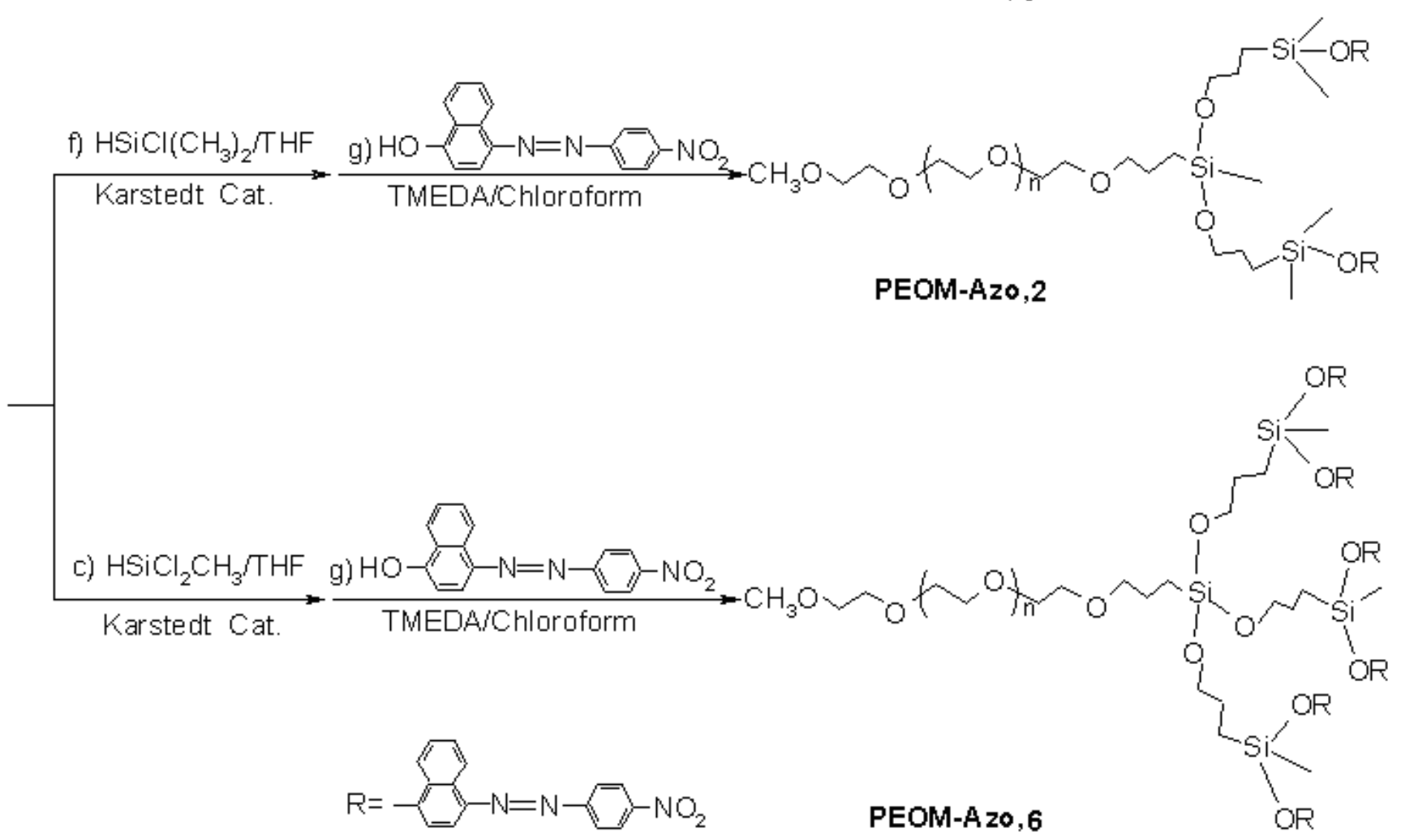

Scheme 1. Synthesis rout of PEOM-Azo, 2 and PEOM-Azo, 6 systems utilizing hydrosilylation-alcoholysis process.

Based on our observation only a very small quantities of karstedt catalyst were sufficient; typically, the amounts were in the region of $10^{-5}$ catalyst $/ \mathrm{CH}=\mathrm{CH}_{2}$, but in some cases the ratio of $10^{-7}$ catalyst $/ \mathrm{CH}=\mathrm{CH}_{2}$ has been used successfully (30). An important key to develop desired compounds is the alcoholysis conditions. Based on our experience, this reaction can be maintained at mild conditions by carefully controlling the dropping speed of alcoholysis reagent. TMEDA as the base and catalyst can facilitate the $\mathrm{SiCl} / \mathrm{OH}$ condensation (22). 
The GPC measurements using polystyrene standards yielded slightly smaller molecular weights presumably due to interaction between the azo-functionalized copolymers and the columns. . Obtaining GPC data for polymers containing chromophore have been already reported $(31,32)$.

\section{Photoisomerization study}

As depicted in Figures $3 \mathrm{a}$ and $3 \mathrm{~b}$, UV irradiation transformed most of the azo moieties from trans into the cis conformation (33-38). It may be due to the flexible carbosiloxane branches connected to azo moieties which prevent stacking between adjacent azo chromophores. Over $120 \mathrm{~min}$ for PEOM-Azo, 2 only a very small proportion of the system in the cis form thermally reverted to the trans form (Figures 3c and 3d), as the absorbance increased merely from 0.37 to 0.40 . In contrast, under visible light the isomerization was almost completed within 5 minutes, with the absorbance increasing to 0.54 and staying at that value with further irradiation. Clearly, under our experimental conditions, isomerization from the cis to the trans conformation was predominantly photoinduced. Therefore, dark incubation of the UV-exposed PEOM-Azo, 2 and PEOM-Azo, 6 resulted in relatively slow thermal cis-trans back isomerization.

\section{Effect of UV irradiation on the size of PEOM- Azo, 2 and PEOM-Azo, 6 systems}

The PEOM-Azo, 2 and PEOM-Azo, 6 carriers were formed by self-aggregation in aqueous media. The azo-groups built compact packing in the core network of aggregates due to a strong non-covalent aromatic-aromatic interaction. Under the irradiation of UV light, the trans azo-groups with a rod-like shape are converted to the their cis ones with bent shape, which could disrupt the original relatively tight packing and bring a loose stacking formation in the aggregates (7).

On the other hand, a temporary change of the conformation because of photoisomerization of azo moiety could have an effect on polarity, hydrophobicity and geometrical shape of the carriers $(9,10)$. With regard to the fact that the trans azo- group is planar, while in the cis azo-group the aromatic rings are not planar so the PEOM-Azo, 2 and PEOM-Azo, 6 systems in cis form occupy more volume than the trans form (35). Thus, the trans-cis isomerization is accompanied by an increase in total occupied volume (Figure 4a) $(7,35,36)$.

\section{Micellar aggregation and CMC determination studies}

Amphiphilic macromolecules with appropriate balance of hydrophilic/hydrophobic segments can form a micellar structure when exposed to a selective solvent (37). The amphiphilic nature of the PEOM-Azo, 2 and PEOM-Azo, 6 as lineardendritic diblock copolymers consisting of hydrophilic PEOM and hydrophobic carbosiloxane blocks, make available to form micelles in water. The hydrophobic chromophore-terminated carbosiloxane segments could make strong noncovalent aromatic-aromatic interaction between Magneson II chromophores. The newly prepared aggregates can be stabilized by peripheral hydrophilic PEOM chains. Accordingly, these amphiphilic macromolecules self-assemble into micelles with hydrophobic chromophor-terminated carbosiloxane segments as the core and hydrophilic PEOM segments and as the corona. The schematic illustration is shown in Figure 1.

During this process, hydrophobic pyrene is incorporated in the interior of the aggregates (38). Therefore, by determining the amount of pyrene solubilized in aqueous solution, $\mathrm{CMC}$ value of PEOM-Azo, 2 and PEOM-Azo, 6 micelles could be determined.

Consequently, as the concentration of PEOMAzo, 2 and PEOM-Azo, 6 micellar solutions increase, the absorbance (A) became more. It is implies that PEOM-Azo, 2 and PEOM-Azo, 6 micellar solutions deeply increase the solubility of pyrene in water through incorporating themselves into the micellar phase. As a result, with increasing PEOM-Azo, 2 and PEOM-Azo, 6 concentration, a point is reached (CMC) when the bulk of water becomes saturated with monomeric PEOM-Azo, 2 and PEOM-Azo, 6 amphiphiles. At this point, any additional enhances in PEOM-Azo, 2 and PEOMAzo, 6 concentrations results to the formation of micelles in the solution (Figure 5a, b, c and d).

These CMC values are lower than those reported for other polymeric micelles and comparable with dendritic micelles (Table 2) (39). This comparison implies a very strong tendency of the lineardendritic diblock copolymers toward micelle formation and their relatively thermodynamically stable entities. This result could be accounted by hydrophobicity of carbosiloxane chains as well as strong non-covalent aromatic-aromatic interaction of chromophores in the core of aggregates (40). 


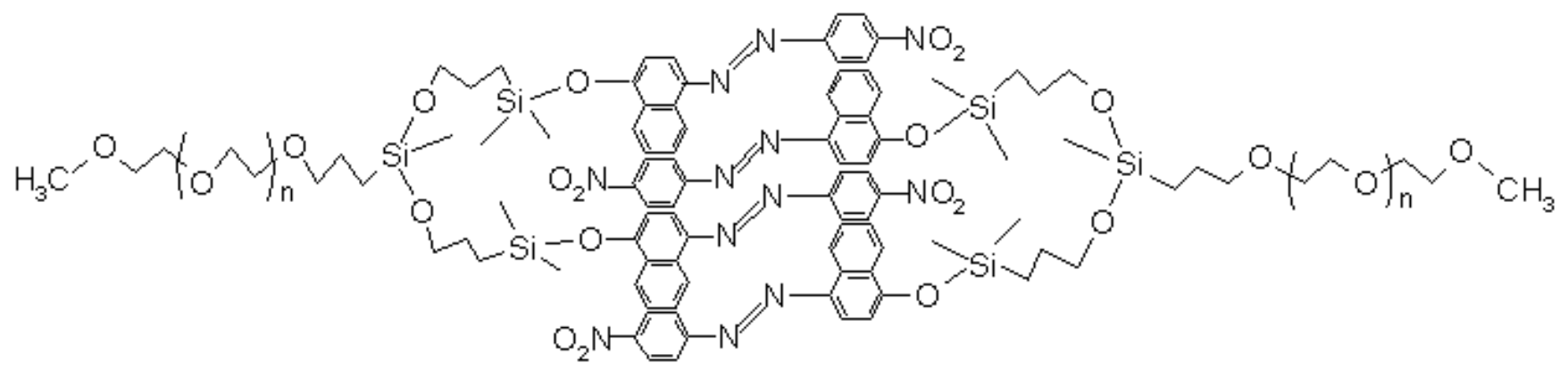

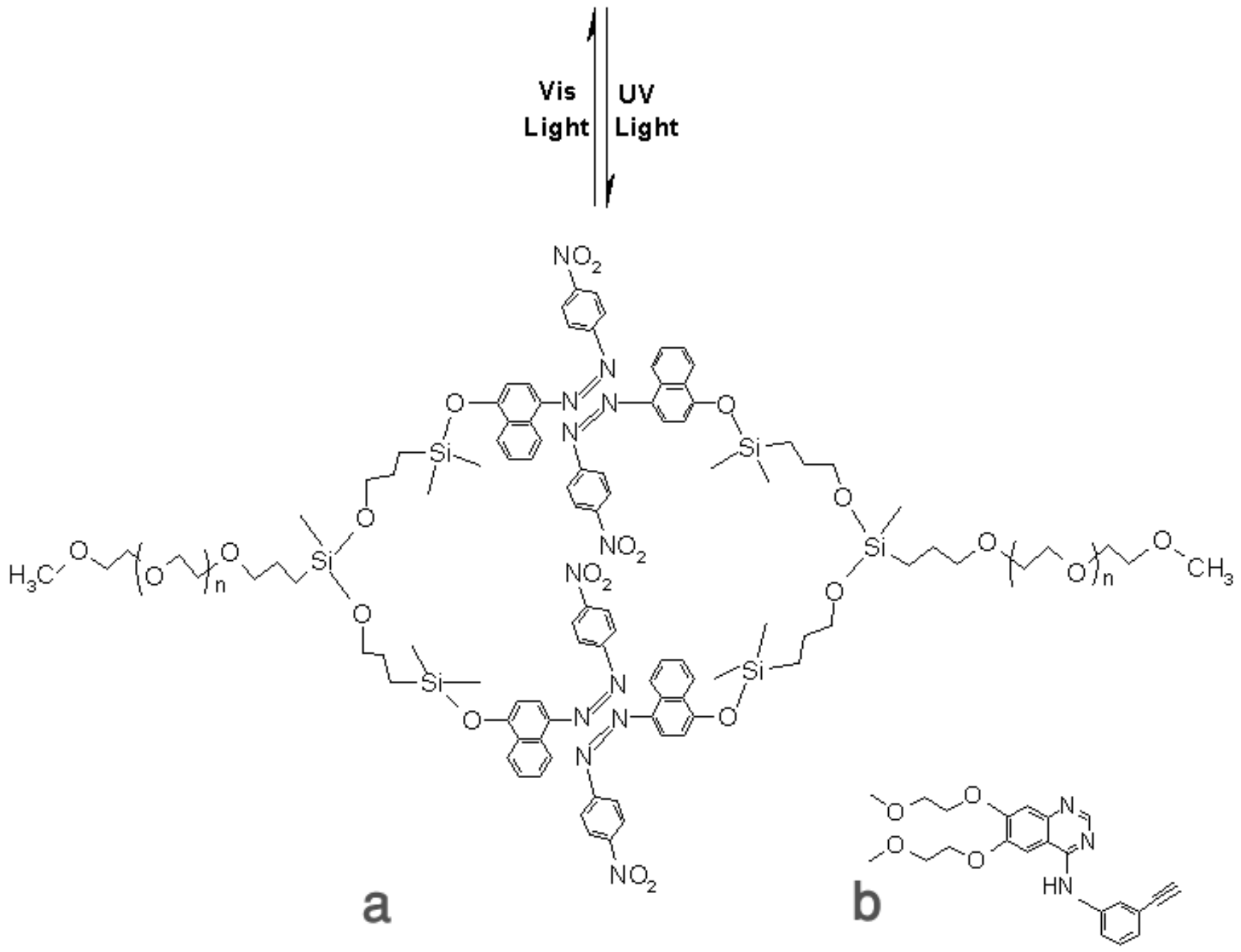

Figure 4. Illustration of UV and visible-light induced isomerization of PEOM-Azo, 2 as a typical example (a) and structure of hydrophobic anti-cancer molecule (erlotinib) as a guest model (b).

\section{Micellar aggregation numbers}

According to the equation, the straight lines of logarithm of the intensity ratio versus the quencher concentration $\mathrm{C}_{\mathrm{q}}$ were plotted (Figure 5e) (27). As shown in this Figure, good linear correlations appear for each PEOM-Azo, 2 and PEOM-Azo, 6 systems. The $\mathrm{N}_{\text {agg }}$ values increase on going from $\mathrm{E}$ form to $\mathrm{Z}$ form as well as it increases in PEOMAzo, 2 isomers relative to PEOM-Azo, 6 counterparts. This phenomenon appears reasonable, regarding to more strong aromatic-aromatic interactions in $\mathrm{E}$ form than $\mathrm{Z}$ form and more quantity of interactions in PEOM-Azo, 6 than PEOM-Azo, 2. The total numbers of Magneson II 
group in the core of each micellar aggregates could be determined using $\mathrm{N}_{\mathrm{agg}}$ values (Table 2).

\section{Evaluation of size and size distribution of the micellar aggregates}

Regarding to DLS data, it seems that size of the unloaded carriers have a dependence on the quantity of the aromatic-aromatic interaction in medium (Figure 4a) (Table 3). Consequently, with increasing the degree of the interaction, the carriers are smaller. For instance the observed decrease in size of PEOM-Azo, 2 carriers in trans form, with higher degree of the interactions, relative to its cis counterpart, with lesser degree of the interactions, is apparently due to greater propensity of the aromatic moieties to form aggregates with compact cores in the medium. A similar experience observed in PEOM-Azo, 6 in trans form when compared to PEOM-Azo, 6 in cis form. Therefore, it appears that the increase in the average size of the carriers due to trans-cis isomerization is reflective of weakened interactions, which results in loosening of the compact core network.

On the other hand, the drug-incorporated carriers of PEOM-Azo, 6 indicate a remarkable enhance in their average size, relative to PEOM-Azo, 2. This result was consistent with the fact that a higher degree of the interactions in core network of PEOM-Azo, 6 than PEOM-Azo, 2 could increase encapsulation leading to formation of bigger particles (38). As could seen in table 3, through comparison of drug-incorporated PEOM-Azo $(2,6)$, for instance, the mean diameters of the unloaded carriers measured by DLS in cis form are 738.0 and $937.8 \mathrm{~nm}$ for PEOM-Azo, 2 and PEOM-Azo, 6, respectively. After incorporation of erlotinib into the carriers the respective mean diameters are 877.6 and $1314.9 \mathrm{~nm}$, as order. The polydispersity factors PDI $\left(\mu_{2} / \Gamma^{2}\right)$ of the PEOM-Azo, 2 and PEOM-Azo, 6 carriers suggested a narrow size distribution in all cases.

\section{Optimization of drug-Incorporation Content (IC) and Loading Efficiency (LE)}

Controlling the amount of incorporated drug to obtain stable drug carriers is imperative, because this quantity is likely to influence micelle's stability (Figure 4b) (39). Therefore, the IC and LE of the drugs in PEOM-Azo, 2 and PEOM-Azo, 6 systems were analyzed in different drug:carrier ratio. In general, as seen drug LE diminishes with increasing ratio of drug:carrier for all of the carriers. The maximum drug LE was obtained with drug:carrier ratios of $0.1: 1$ and $0.2: 1$ for trans and cis forms, respectively. These results obviously indicated that the lower drug:carrier ratios, e.g., 0.1:1.0 and 0.2:1.0, cause a considerable enhance in drug IC and LE for PEOM-Azo, 2 and PEOM-Azo, 6 systems (Table 4). The acquired data are in agreement with other reports in the similar drug:carrier ratios $(43,44)$.

In contrast, a large amount of material was precipitated with drug:carrier ratios more than $0.8: 1.0$. It seems that the incorporation of drugs into the carriers competes with their precipitation in the solvent when their ratio increased, resulting to lowered drug IC and LE (Table 4) (43). In simple terms, the guest molecules interact with each other more strongly than with the PEOM-Azo, 2 and PEOM-Azo, 6 systems, especially for higher drug:carrier ratios. Thus, the drug:carrier ratio is a key parameter in which appreciably controls the drug loading during the drug-incorporation processes. Again, higher hydrophobic drug (erlotinib) IC was obtained with PEOM-Azo, 6 than PEOM-Azo, 2. It could be explained by the reason that PEOM-Azo, 6 contains larger blocks of carbosiloxane branches bearing azo chromospheres as the hydrophobic portion. Therefore, the hydrophobic drug could be encapsulated in network's cavities of PEOM-Azo, 6 more effective than that of PEOM-Azo, 2. As reported, by Yokoyama et al., hydrophobic interactions among a hydrophobic block, may be an important key to controlling carriers in drug loading process (43). A reverse effect, vice versa, was observed by hydrophilic drugs (erlotinib hydrochloride). Our results showed a higher drug IC to somewhat by using PEOM-Azo, 2 in trans form than that of PEOM-Azo, 6. It might be justified because IC and LE for hydrophilic drug are highly dependent on the weight fraction of hydrophilic segment in amphiphilic polymers.

In the case of the hydrophobic drugsincorporation into PEOM-Azo, 2 and PEOM-Azo, 6 in cis form, upon UV irradiation photoisomerization could decrease the amount of trans azo-groups and simultaneously increase the cis azo-groups in the core of carriers. However some of the trans azogroups are still present, although most of them are in cis conformation, which could cause aromaticaromatic interactions with hydrophobic drugs. This 
is consistent with the fact that existence of these interactions in core network enhances encapsulation of hydrophobic guest molecules (44).

Interestingly, IC in this case is even more than that of PEOM-Azo, 2 and PEOM-Azo, 6 in trans form. As was presented in Table 4, ICs are 2.6 and 4.83 by ratio of $0.1: 1.0$ for PEOM-Azo, 2 in trans and cis forms, respectively. It could be attributed to the more open structure of cis form relative to trans form, owing to loosening of the compact core in the network of cis form which allows more encapsulation.

On the other hand, the low amounts of IC of PEOM-Azo, 2 and PEOM-Azo, 6 in trans form during the incorporation of hydrophilic drug (erlotinib hydrochloride) are implying another phenomenon. For instance, IC is 0.6 and 0.5 , by ratio of 0.1:1.0 for PEOM-Azo, 2 and PEOM-Azo, 6 , respectively. It is natural to think that most of hydrophilic drug was entrapped in the hydrophilic exterior of the carriers (as pointed out in upmentioned section).

\section{Evaluation of in vitro release kinetics}

As it is well-known, stability of the micellar system in blood or serum conditions is a key factor due to the more destabilizing conditions therein as compared to that of aqueous buffers. In this context, as the first step one must study the stability and hydrolytic behavior of special kinds of aggregates of PEOM-Azo, 2 and PEOM-Azo, 6 in trans and cis forms. It was shown that the loading efficiency (LE) for of trans (low polarity) and cis (high polarity) in the above-mentioned carriers were different from each other. Meanwhile, in order to test this hypothesis that the carriers could load hydrophilic drug in cis form and vice versa, we designed several experiments in various conditions as follows:

\section{Release of erlotinib from PEOM-Azo, 2 and PEOM-Azo, 6 during $U V$ irradiation exposure after incorporation}

As previously mentioned, release behavior of the carriers under UV irradiation was studied in order to find out the effect of light as an external stimulus on the third step of a controlled release system. As shown in Figure $7 \mathrm{a}$, the release of erlotinib from PEOM-Azo, 2 and PEOM-Azo, 6 in trans form when exposed to UV irradiation after incorporation, revealed similar behavior with its counterpart outside the UV irradiation (both at the ratio $0.1: 1.0)$. However, the release rate of the former is faster than the latter. It is speculated that the guest molecule is physically entrapped in core of the carrier, as well as the release of the guest molecule from carriers occurs mainly through diffusion. With trans-cis isomerization in the PEOM-Azo, 2 and PEOM-Azo, 6 systems, the hydrophobic aromaticaromatic interactions of trans azo-groups decrease with simultaneous expansion of the inner hydrophobic cavities owing to generating cis azogroups. With keeping this in mind, it is reasonable to accept that phtoisomerization could result in increasing of diffusion rate of the drug through the carrier.

Release of erlotinib from PEOM-Azo, 2 and PEOM-Azo, 6 in trans form

At the first 30 Min, less than $20 \%$ and $15 \%$ of erlotinib was released burstly for PEOM-Azo 2 and PEOM-Azo 6 in trans form, respectively. It might be due to the diffusion of the drug that was poorly entrapped in the exterior part, of carriers. Then, a sustained release of the hydrophobic drug had been observed at a slower release rate. The hydrophobic interaction involving the drug and the hydrophobic core of the carriers is a main reason leading to a controlled release rate for the carriers. Notably, drug release from PEOM-Azo 6 carrier was slower than from that of PEOM-Azo 2 (Figure 7b). The observed result can be explained by the higher drug IC in PEOM-Azo 6 carrier $(5.24$, by ratio of $0.1: 1.0)$ relative to drug IC in PEOM-Azo 2 (2.62, by ratio of $0.1: 1.0$ ). The higher drug IC within the carriers is supposed to increase interactions between the hydrophobic drugs and the hydrophobic portions of the PEOM-Azo, 2 and PEOM-Azo, 6 which decreases drugs release rate (45).

\section{Release of erlotinib from PEOM-Azo, 2 and PEOM-Azo, 6 in cis form}

As previously mentioned, the compact cores of carriers in $\mathrm{Z}$ form have been slightly loosened. As a result, the inner cavities of carriers are enlarged to some what. This further leads to faster rate of diffusion of the encapsulated species from the interior pockets of the network. Comparison of release of erlotinib-incorporated PEOM-Azo, 2 systems in $\mathrm{E}$ and $\mathrm{Z}$ form reveals that while the 
carriers of $E$ form permit the optimum controlled release, in the latter, the carriers of $\mathrm{Z}$ form display a higher release rate (Figure $7 b$ ).

\section{Release of erlotinib hydrochloride from PEOM- Azo, 2 and PEOM-Azo, 6 in trans form}

In this case, the burst release was near to $38 \%$ during the first 60 Min (Figure 7c). It was the highest burst release among the all experiments. Relating to the low amount of IC (by ratio of $0.1: 1.0 ; 0.6$ and 0.5 for PEOM-Azo, 2 and PEOMAzo, 6, respectively), most of hydrophilic drug was entrapped in the exterior of the carriers.

\section{Release of erlotinib hydrochloride from PEOM- Azo, 2 and PEOM-Azo, 6 in cis form}

Our results revealed that the hydrophilic drug have approximately similar release kinetics to hydrophobic drug. However, it is released faster than hydrophobic one having further burst release to some what. A possible explanation is that the hydrophilic drug, conversely to hydrophobic one, could be encapsulated both into the exterior and interior part of the carriers. Thus, at first, the hydrophilic drug in the hydrophilic exterior shell of carrier start to release with a fast rate and then the hydrophilic drug incorporated in the interior part is gradually released (Figure 7c). The incorporation of hydrophilic drugs into the core of the carriers possibly could be ascribed to more polar entity of them containing cis azo-groups which tend to attract hydrophilic drugs (46).

\section{CONCLUSION}

In this study, a new type of aggregates has been developed concerning polarity as PEOM-Azo, 2 and PEOM-Azo, 6 in trans and cis forms. The Loading Efficiency for both of trans, low polarity, and cis, high polarity, in the above-mentioned carriers would be different. For incorporation of drug into the respective carriers from this polymeric system, the drug:carrier ratio significantly influenced drug-incorporation content and loading efficiency. The incorporation of drug into the carriers in various drug:carrier ratios $(0.1: 1.0$, $0.2: 1.0,0.4: 1.0$ and $0.8: 1.0$ ) were optimized in which the best ratio of LE were obtained as 0.1:1.0 and 0.2:1.0 for trans and cis forms, respectively. It was shown that the incorporation content and in vitro release behavior of the carriers is influenced by their photoisomerization properties. Consequently, it could regulate in turn, hydrophilic and hydrophobic characteristics of the carriers. Finally, the findings from this study highlight the potential of a new delivery vehicle for sustained release of drug molecules from the matrix of carriers.

\section{ACKNOWLEDGEMENT}

The authors gratefully acknowledge University of Tabriz for the financial supports of this project.

\section{REFERENCES}

1. Pires A, Fortuna A, Alves G, Falcão A. Intranasal Drug Delivery: How, why and what for?. J Pharm Pharmaceut Sci, 2009;12:288-311.

2. Nasongkla N, Bey E, Ren J, Ai H, Khemtong C, Guthi JS, Chin S-F, Sherry AD, Boothman DA, Gao J. Multifunctional polymeric micelles as cancer-targeted, MRI-ultrasensitive drug delivery systems. Nano Lett, 2006; 6:2427-3240.

3. Chung JE, Yokoyama M, Okano T. Inner core segment design for drug delivery control of thermo responsive polymeric micelles. J Control Release, 2000; 65:93-103.

4. Laura J, Waters V, Evangelos V. In vitro controlled drug release from loaded microspheres-dose regulation through formulation. J Pharm Pharmaceut Sci, 2007;10:464-472.

5. Gupta U, Bharat H, Agashe, Jain NK. Polypropylene imine .dendrimer mediated solubility enhancement: effect of $\mathrm{pH}$ and functional groups of hydrophobes. J Pharm Pharmaceut Sci, 2007;10:358-367.

6. Qin S, Geng Y, Discher DE, Yang S. Temperaturecontrolled assembly and release from polymer vesicles of poly(ethyleneoxide)-block- poly(Nisopropylacrylamide). Adv Mater, 2006; 18:29052909.

7. Liu X, Jiang M. Optical switching of self-assembly: micellization and micelle-hollow-sphere transition of hydrogen-bonded polymers. Angew Chem Int Ed, 2006; 45:3846-3850.

8. Zhao Y. Rational design of light-controllable polymer micelles. The Chemical Record, 2007; 7: 286-294.

9. Ishihara K, Namada N, Kato S, Shinohara I. Photoinduced swelling control of amphiphilic azoaromatic polymer membrane. J Polym Sci A: Polym Chem, 1984; 22:121-128.

10. Su W, Luo Y, Yan Q, Wu S, Han K, Zhang Q, Gu $\mathrm{Y}$, Li Y. Photoinduced fusion of micro-vesicles self-assembled from azobenzene-containing 
amphiphilic diblock copolymers. Macromol Rapid Commun, 2007; 28:1251-1256.

11. Van den Mooter G, Kinget R. Oral colon-specific drug delivery: a review. Drug Delivery, 1995; 2:8193.

12. Schacht E, Gevaert A, Kenawy E, Koen M, Willy V, Peter A, Robert C. Polymers for colon specific drug delivery, J Control Release, 1996; 39:327-338.

13. Han M, Hara M. Fluorescence enhancement from self-assembled aggregates: substituent effects on self-assembly of azobenzenes. J Am Chem Soc, 2005; 127:10951-1055.

14. Barrio J. D, Oriol L, Sánchez C, Serrano J. L, Cicco A. D, Keller P, Li M-H. Self-Assembly of LinearDendritic Diblock Copolymers: From Nanofibers to Polymersomes J Am Chem Soc, 2010;132:37623769.

15. Namazi H, Jafarirad S. Synthesis of block and grafted copolymers containing spacer-linked chromophore based on cellulose and polyethylene glycol. J Appl Polym Sci, 2004; 94:1175-1185.

16. Namazi H, Jafarirad S. Preparation of the new derivatives of cellulose and oligomeric species of cellulose containing magneson II chromophore. J Appl Polym Sci, 2008; 110:4034-4039.

17. Didehban K, Namazi H, Entezami AA. Dendrimerbased hydrogen-bonded liquid crystalline complexes: Synthesis and characterization. Europ Polym J, 2009; 45:1836-1344.

18. Namazi H, Adeli M, Zarnegar Z, Jafarirad S, Dadkhah A, Shukla A. Encapsulation of nanoparticles using linear-dendritic macromolecules. Colloid Polym Sci, 2007; 285:1527-1533.

19. Didehban K, Namazi H, Entezami AA., Synthesis and characterization of liquid crystalline diethanolamine-based dendrimers. Polym Adv Technol, 2009; 20:1127-1135.

20. Namazi H, Adeli M. Dendrimers of citric acid and poly (ethylene glycol) as the new drug-delivery agents. Biomaterials, 2005; 26:1175-1183.

21. Namazi H, Adeli M. Synthesis of barbell-like triblock copolymers, dendritic triazine-blockpoly(ethyleneglycol)-block-dendritic triazine and investigation of their solution behaviors. J Polym Sci A: Polym Chem, 2005; 43:28-41.

22. Son HJ, Han WS, Kim H, Kim CKJ, Lee C, Kang SO. End-capped silole dendrimers on a carbosilane periphery: potential electroluminescent materials. Organometallics, 2006; 25:766-774.

23. Namazi H, Jafarirad S. Application of Hybrid Organic/Inorganic Dendritic ABA Type Triblock Copolymers as New Nanocarriers in Drug Delivery Systems (accepted 2 october 2010).

24. Malik N, Wiwattanapatapee R, Klopsch R, Lorenz, K, Frey H, Weener JW, Meijer EW, Paulus W,
Duncan, R. Dendrimers: Relationship Between Structure and Biocompatibility in Vitro, and Preliminary Studies on the Biodistribution of 125ILabelled Polyamidoamine Dendrimers in Vivo. J Control Release, 2000; 65: 133-148.

25. Namazi H, Jafarirad S. Hybrid organic/inorganic dendritic triblock copolymers: Synthesis, nanostructure characterization, and micellar behavior. J Appl Polym Sci, 2010; 117:1085-1094.

26. Wang L, Weber WP. Synthesis and properties of novel comb polymers: unsaturated. carbosilane polymers with pendant oligo(oxyethylene) groups. Macromolecules, 1993; 26:969-974.

27. Turro NJ, Yekta A. Luminescent probes for detergent solutions: a simple procedure for determination of the mean aggregation number of micelles. J Am Chem Soc, 1978; 100:5951-5952.

28. Zhao GX, Zhu BY, Principles of Surfactant Action. China Light Industry Press, Beijing, 2003.

29. Hu Y, Jiang X, Ding Y, Zhang L, Yang C, Zhang J, Chen J, Yang Y. Preparation and drug release behaviors of nimodipine - loaded poly (caprolactone) - poly (ethyleneoxide) - polylactide amphiphilic copolymer nanoparticles. Biomaterials, 2003; 24:2395-2404.

30. Speier J, Webster J, Barnes GH. The use of group VIII metal catalysts. J Am Chem Soc 79:974-979.

31. Jiang J, Tong X, Morris D, Zhao Y. Toward photocontrolled release using light-dissociable block copolymer micelles. Macromolecules, 39:4633-4640.

32. Cui L, Lattermann G. Synthesis and characterization of new acrylate and methacrylate monomers with pendant pyridine groups. Macromo Chem Phys, 203:2432-2437.

33. Suzuki, H., Electronic Absorption Spectra and Geometry of Organic Molecules. Academic Press, New York, 1967.

34. Griffiths J. Photochemistry of azobenzenes and its derivatives. J Chem Soc Rev, 1972; 1:481-493.

35. Kumar G, Neckers D. Photochemistry of Azobenzene-Containing Polymers. Chem Rev, 1985; 89:1915-1925.

36. Norman L, Barrett C. Solution properties of selfassembled amphiphilic copolymers determined by isomerization spectroscopy. J Phys Chem B 2002; 106:8499-8503.

37. Zhou Z, Chu B. Phase behavior and association properties of poly(oxypropylene)poly(oxyethylene)-poly(oxypropylene) triblock copolymer in aqueous solution. Macromolecules, 1994; 27:2025-2033.

38. Riley T, Heald CR, Stolnik S, Garnett MC, Illum L, Davis SS. Core-shell structure of PLA-PEG nanoparticles used for drug delivery. Langmuir, $2003 ; 19: 8428-8435$. 
39. Ihre HR, Padilla De Jesus OL, Szoka FCJ, Fréchet JMJ. Polyester dendritic systems for drug delivery applications: design, synthesis, and characterization. Bioconjugate Chem, 2002; 13:443- 452.

40. Sunder A, Kramer R, Hanselmann R, Mulhaupt R, Frey H. Molecular nanocapsules based on amphiphilic hyperbranched polyglycerols. Angew Chem Int Ed Engl, 1999; 38:3552-3554.

41. $\mathrm{Xu} \mathrm{Y,} \mathrm{Du} \mathrm{Y,} \mathrm{Huang} \mathrm{R,} \mathrm{Gao} \mathrm{L.} \mathrm{Preparation} \mathrm{and}$ modification of N-(2-hydroxyl) propyl-3-trimethyl ammonium. Biomaterials, 2003; 24:5015-5022.

42. Lin W, Juang L, Lin C. Stability and release performance of a series of pegylated copolymeric micelles. Pharm. Res, 2003; 20:668-673.

43. Yokoyama M, Satoh A, Sakurai Y, Okano T, Matsumura Y, Kakizoe T, Kataoka K.
Incorporation of water - insoluble anticancer drug into polymeric micelles and control of their particle size. Journal Control Rel, 1998; 55:219229.

44. Yu B, Okano T, Kataoka K, Kwon G. Polymeric micelles for drug delivery: solubilization and haemolytic activity of amphotericin B. J Control Rel, 1998; 53:131-136.

45. Chakraborty H, Banerjee R, Sarkar M. Incorporation of NSAIDs in micelles: implication of structural switchover in drug-membrane interaction. Biophys Chem, 2003; 104:315-325.

46. Shiraishi K, Kawano K, Minowa T, Maitani Y, Yokoyama M. Preparation and in vivo imaging of PEG-poly(L-lysine)-based polymeric micelle MRI contrast agents. J Control Rel, 2009; 136:14- 20. 